\title{
An Improved Protocol for the Virtual Screening Discovery of Novel Histone Deacetylase Inhibitors
}

\author{
Qiuhang Song, ${ }^{a}$ Tingting Liu, ${ }^{a}$ Yucui Liu, ${ }^{b}$ Shuyue Wang,${ }^{a}$ Cong Fan, ${ }^{c}$ Lihua Zheng, ${ }^{d}$ \\ Yongli Bao, ${ }^{a}$ Luguo Sun, ${ }^{a}$ Chunlei Yu, ${ }^{d}$ Ying Sun, ${ }^{a}$ Zhenbo Song,${ }^{a}$ Guannan Wang, ${ }^{d}$ \\ Yanxin Huang, ${ }^{* a}$ and Yuxin $\mathrm{Li}^{*}, d$ \\ ${ }^{a}$ National Engineering Laboratory for Druggable Gene and Protein Screening, Northeast Normal University; \\ Changchun 130024, China: ${ }^{b}$ State Key Laboratory of Microbial Technology, Shandong University; Qingdao 266237, \\ China: ' Sun Yat-Sen Memorial Hospital, Sun Yat-Sen University; Zhongshan 510275, China: and ${ }^{d}$ Research Center \\ of Agriculture and Medicine gene Engineering of Ministry of Education, Northeast Normal University; Changchun \\ 130024, China.
}

Received April 14, 2019; accepted July 5, 2019; advance publication released online August 9, 2019

\begin{abstract}
Histone deacetylases (HDACs) are enzymes that play a key role in structural modification and gene expression. The overexpression of HDAC is associated with cancer, and thus inhibiting the enzyme could be an efficient cancer therapy. To discover new HDAC inhibitors (HDACis), we proposed an improved protocol combining a hierarchical pharmacophore search, molecular docking, and molecular dynamic simulations. The test results showed that the improved screening protocol effectively reduced the false-positive rates of drug-like chemicals. Based on the protocol, we obtained 16 hit compounds as potential HDACis from the Life Chemicals database. Enzyme inhibition experiments showed that two of the hit chemical compounds had HDAC-inhibitory effects. In vitro assays showed that Z165155756 could selectively inhibit the proliferation of cancer cells and specifically promoted apoptosis and induced G1/S phase arrest in A2780 cells. It may have potential therapeutic effects in ovarian cancer and is worthy of further investigation.
\end{abstract}

Key words histone deacetylase inhibitor; pharmacophore; docking; molecular dynamic simulation; ovarian cancer

\section{Introduction}

Histone deacetylases (HDACs) can remodel chromatin and play an important role in regulating gene expression. ${ }^{1)}$ To date, based on HDAC sequence similarity to yeast orthologues Rpd3, Hdal, and Sir2, the HDAC family is divided into four classes: class I (HDAC1-3, and -8); class II (HDAC4-7, 9, and 10); class III (sirtuins 1-7); and class IV (HDAC11). ${ }^{2}$ Class I, II, and IV HDACs are classical $\mathrm{Zn}^{2+}$-dependent enzymes harboring a catalytic pocket with $\mathrm{Zn}^{2+}$-chelating compounds. ${ }^{3)}$ Overexpression of HDACs in various cancers may inhibit the expression of growth-suppressive genes (p21 and p27) that play an important role in promoting cancer cell proliferation. ${ }^{4)}$ HDAC inhibition has therefore become an important target for cancer therapy. ${ }^{5)}$ In the expanding field of anticancer drugs, HDAC inhibitors (HDACis) are playing an increasingly important role. ${ }^{6}$ At present, vorinostat (SAHA), romidepsin (FK228), belinostat (PXD-101), panobinostat (LBH589), and chidamide are clinically used in treating cutaneous T-cell lymphomas, multiple myeloma, and peripheral T-cell lymphoma.') However, dose-limiting toxicities including thrombocytopenia, arrhythmia, gastric side effects, nausea, and fatigue are observed with the administration of these HDACis. ${ }^{8}$ Novel HDACis with fewer side effects are urgently needed.

High-throughput screening of large chemical compound databases has been a primary source for the identification of novel lead chemical compounds. ${ }^{9)}$ Virtual screening is a powerful method used for the rapid discovery of novel, original chemical candidate compounds from chemical databases like that of Life Chemicals (www.lifechemicals.com). ${ }^{10)}$ The method has also been used to identify many HDACis with novel structures. ${ }^{11,12)}$ Currently, pharmacophore-based studies, molecular docking, and molecular dynamic (MD) simulation approaches are used independently or in combination to evaluate effective inhibitors. ${ }^{12)}$ Based on our research results, we present an improved protocol for the discovery of novel HDACis involving screening of different structure-based pharmacophore models and docking into the active site of HDAC8 to find drug-like compounds in the Life Chemicals database. Complexes of compounds with high docking scores and HDAC8 are then subjected to MD simulation, followed by analysis of the interactions of these compounds with HDAC and their free energies $\left(\triangle G_{\text {bind }}\right)$ to identify potential HDACis. ${ }^{13)}$

After the suite of screening procedures, 16 compounds with high $\triangle G_{\text {bind }}$ scores were identified. Subsequent enzyme activity experiments using an HDACi screening kit indicated that 2 of the 16 candidates had HDAC-inhibitory effects. In vitro assays indicated that Z165155756 induces apoptosis and inhibits the proliferation of several types of cancer cells, especially of A2780 cells. The improved protocol for the virtual screening discovery of novel HDACis led to the identification of the chemical candidate Z165155756, which is a promising HDACi, has potential therapeutic effects in ovarian cancer, and is worthy of further investigation.

\section{Experimental}

Pharmacophore Modeling We used the GALAHAD program in SYBYL-X 2.0 for ligand-based pharmacophore modeling, after which 7 HDACis with different structures were selected as representative chemical compounds. Most of the 
parameters used the default values, with the activity column of $\mathrm{pIC}_{50}$, the exception of 100 generations, and keeping the best model of 20 . At least 5 of 7 features in the pharmacophore model had to match. Finally, UNITY in SYBYL-X2.0 was used for flex searches to screen the Life Chemicals candidate database.

Molecular Docking (MD) GOLD 5.2 software and HDAC8 (PDB ID: 1T69) were selected as the molecular docking screening method and the docking target, respectively. Except for the value of the genetic algorithm (GA) using 30, other parameters were kept as defaults. The top three scoring structures of every ligand were retained at the end of the calculation. When GOLD showed a docking conformation through a specific algorithm, a scoring function was needed to evaluate the ligand receptor affinity. The scoring function took into account the ligand and the receptor of every force, such as salt bridges, hydrogen bonds, hydrophobic stacking forces, etc. In our experiments, we used ChemPLP and ChemScore as the fitness functions for screening.

MD Simulation and Molecular Mechanics-Poisson Boltzmann Surface Area Calculations We used the cocrystallized or the docked binding orientation structures for MD simulation, and the MD simulations of the compounds were carried out using the GROMACS software. We chose the general AMBER force field (AMBER03) and the standard AMBER force field separately for the ligands and protein (1T69). Then the protein and ligands were immersed in a truncated hexahedron box of water (TIP3P) with a margin of $10 \AA$ along each dimension, and counterions were used to neutralize the system. Finally, all the simulations were analyzed under periodic boundary conditions, with all the parameter settings in the MD simulations similar to those reported by Wang et $a l .{ }^{14)}$ The binding free energies were derived using the molecular mechanics-Poisson Boltzmann surface area (MMPBSA) method integrated in $\mathrm{g}$ mmpbsa. ${ }^{15}$ )

HDAC Inhibitory Effect Assay An HDACis screening kit (APExBIO, Houston, TX, U.S.A.) was used to detect the inhibitory effects of the screened candidate chemical compounds. For positive controls or candidate inhibitors, after dissolving Trichostatin A (TSA) or candidate compounds into the appropriate solvent, diluting to 2-fold the desired test concentration with double-distilled water, and adding $50 \mu \mathrm{L}$ of the diluted candidate inhibitor into wells, the concentration of a U.S. Food and Drug Administration-approved HDACi (trichostatin A) or candidate compounds were 20 and $100 \mu \mathrm{M}$, respectively. Then the chemical compounds, HDAC fluorometric substrate (with an acetylated lysine side chain), assay buffer, and HeLa nuclear extracts were placed in 96-well plates and incubated at $37^{\circ} \mathrm{C}$ for $25 \mathrm{~min}$. Lysine developer was then added, and the mixture was incubated for another $30 \mathrm{~min}$ to stop the reaction. The optical density (OD) value was finally calculated as: (OD value of test $\mathrm{HDACi} / \mathrm{OD}$ value of water) $\times 100 \%$.

Cell Lines and Cell Culture The human hepatocellular carcinoma cell line HepG2, human ovarian carcinoma cell line A2780, normal human liver cell line L02, and human ovarian surface epithelial cell line (HOSEpiC) were obtained from the Cell Bank of the Chinese Academy of Sciences (Shanghai, P. R. China). All cells were cultured with Dulbecco's modified Eagle's medium (DMEM) supplemented with 10\% fetal bovine serum (Biyuntian, Hangzhou, P. R. China), 100 U/mL penicillin, and $100 \mathrm{mg} / \mathrm{mL}$ streptomycin (Roche, Upper Ba- varia, Germany) at $37^{\circ} \mathrm{C}$ under a $5 \% \mathrm{CO}_{2}$ atmosphere.

3-(4,5-Dimethylthiazol-2-yl)-2,5-diphenyltetrazolium Bromide (MTT) Assay The MTT assay was used to analyze cell viability after treatment with the chemical candidates. First, cells were plated into 96 -well plates $\left(1 \times 10^{4}\right.$ cells/ well), cultured for $24 \mathrm{~h}$, and then dimethyl sulfoxide (DMSO) or $0,1,10,50$, or $100 \mu \mathrm{M}$ of candidate compounds in the presence of $3 \%$ serum was added to the cultures. After $44 \mathrm{~h}$ of culture, $20 \mu \mathrm{L}$ of MTT $(5 \mathrm{mg} / \mathrm{mL})$ was added to each well for an additional 4-h incubation. The formazan precipitate was dissolved with $100 \mu \mathrm{L}$ DMSO, and finally a micro-enzymelinked immunosorbent assay (ELISA) reader was used to measure inhibition of cell viability at $570 \mathrm{~nm}$. The inhibition ratio was expressed as: (1-OD value of compounds/OD value of control) $\times 100 \%{ }^{16)}$

Cell Cycle Arrest and Cell Apoptosis Analysis To quantify cell cycle arrest and cell apoptosis, A2780 cells were seeded onto 6 -well plates and cultured for $12 \mathrm{~h}$ and then incubated with $40 \mu \mathrm{M}$ of DMSO for $48 \mathrm{~h}$ or with candidate inhibitors for 24, 36, and $48 \mathrm{~h}$. Then, the cells were centrifuged and stained with $5 \mu \mathrm{L}$ fluorescein isothiocyanate (FITC)-Annexin $\mathrm{V}$ and $5 \mu \mathrm{L}$ PI (Biyuntian) for $20 \mathrm{~min}$ or fixed in $75 \%$ alcohol at $4^{\circ} \mathrm{C}$ for $4 \mathrm{~h}$, followed by the addition of RNase and staining with PI (Biyuntian) for $20 \mathrm{~min}$. Cell cycle arrest and apoptosis rates were then measured using flow cytometry (BD FACSCant, Becton Dickinson, NK, U.S.A.) and analyzed using Modfit software (Verity Software House, Topsham, ME, U.S.A.).

Statistical Analysis The experimental results are expressed as mean \pm standard deviation (S.D.) HDAC activity was normalized to the level of DMSO control activity. All experiments were repeated at least three times, and statistical analyses of the data were performed using Student's $t$-test.

\section{Results}

Pharmacophore-Based Virtual Screening We used pharmacophore modeling methods to extract the steric and electronic features of ligand-receptor interactions and then used those features for rapid screening of the Life Chemicals database. $^{10)}$ Seven known HDACis from the literature were used to generate 20 pharmacophore models with GALAHAD, and the top 5 models were chosen for database screening ${ }^{17}$ (Supplementary Table 1). All models derived from more than six ligands (N_NITS $\geq 6$ and FEATS $\geq 6$ ), representing a different trade-off among the conflicting demands of maximizing steric consensus (STERICS), maximizing pharmacophore consensus (HBOND), and minimizing energy (ENERGY) (Supplementary Table 2). We used the top five models, MODEL_011, MODEL_002, MODEL_018, MODEL_014, and MODEL_007, to validate their screening abilities. The five pharmacophore models were used to screen two databases: the HDAC8 inhibitor dataset, which contains 39 active inhibitors and 1,521 unbiased decoys; and the Maximal Unbiased Benchmarking Data Sets for HDACs (MUBD-HDACs), ${ }^{16)}$ which contain all the classical HDACs, including 631 HDACis and 24,609 unbiased decoys. Parameters such as the hit list $(\mathrm{Ht})$, active hits $(\mathrm{Ha})$, and enrichment factor $(\mathrm{EF})$ were used to calculated the pharmacophore model. The results indicated that the top three models with the best EF values were MODEL_002, MODEL_011, and MODEL_018 (Supplementary Table 3). MODEL_002 and MODEL_011 consist of 6 
A
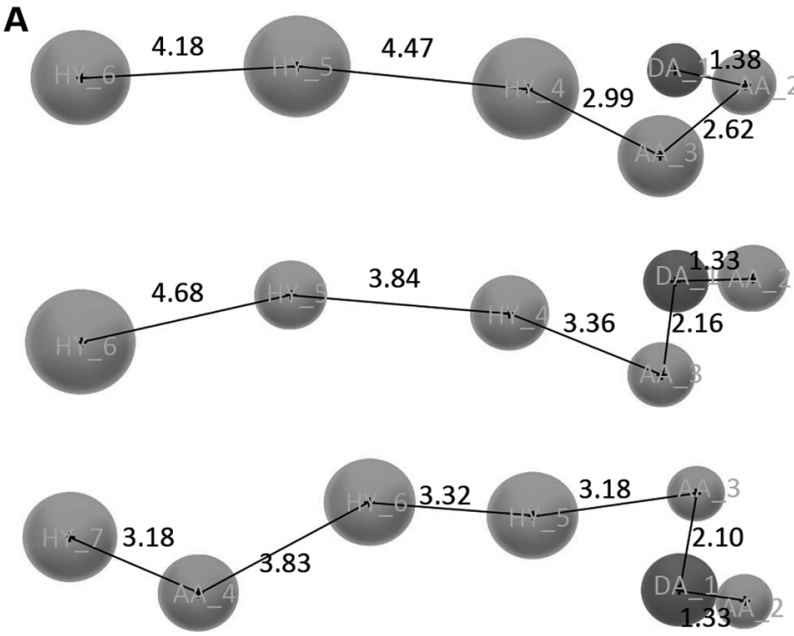

B

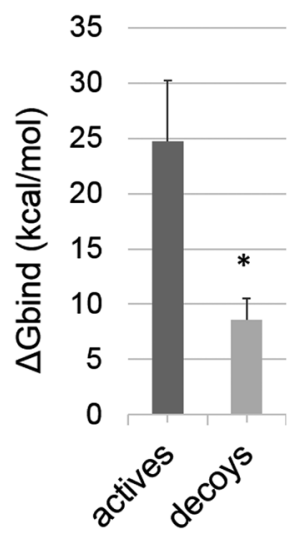

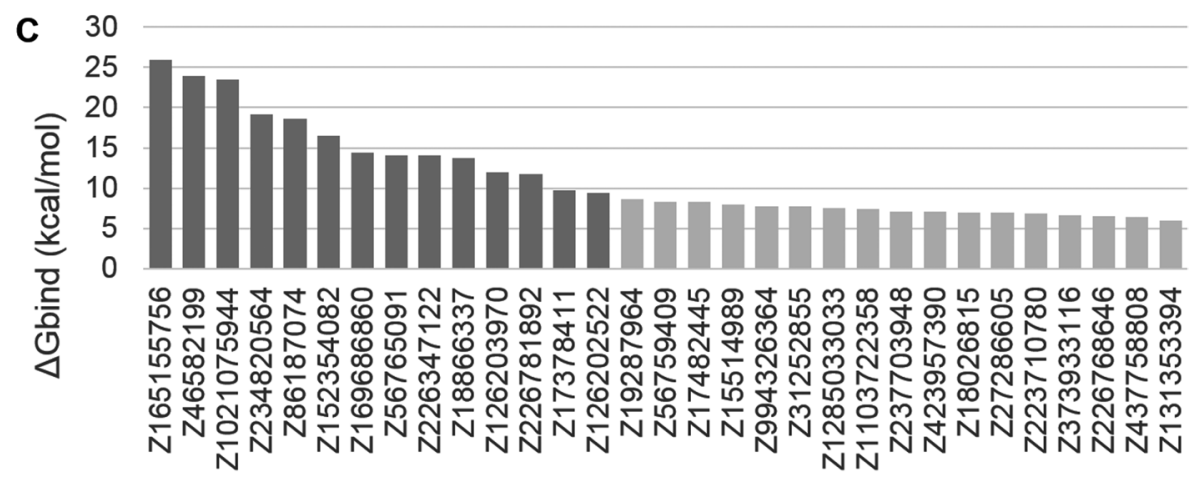

Fig. 1. Generated Pharmacophore Model and $\triangle G_{\text {bind }}$ Values of Candidate Chemical Compounds

(A) Pharmacophore models of MODEL 002 and MODEL 011 (length unit: angstrom). Both have three hydrophobes (HY4, HY5, and HY6), two hydrogen bond (HB) acceptors (AA3 and AA2), and an HB donor (DA1). The pharmacophore model of MODEL 018 has three hydrophobes (HY5, HY6, and HY7), three hydrogen bond (HB) acceptors (AA3, AA4, and AA8) and an HB donor (DA1). Gray spheres, hydrophobes; light gray spheres, HB acceptors; dark gray spheres, HB donors. (B, C) g_mmpbsa was used for calculating the $\triangle G_{\text {bind }}$ values of compounds and HDAC8. Light gray bars, active compounds; white gray bars, inactive compounds. $* p<0.05$, Student's $t$-test.

pharmacophore features, three hydrophobes (HY4, HY5, and HY6), two hydrogen bond (HB) acceptors (AA3 and AA2), and an HB donor (DA1). MODEL_018 consists of three hydrophobes (HY5, HY6, and HY7), three HB acceptors (AA3, AA4, and AA8), and one HB donor (DA1) (Fig. 1A). All the models contain the same pharmacophoric features, but MODEL_018 has an additional HB acceptor feature. Although the chemical features of the models are similar, they differ in their 3D structures, and the differences are considered to be the major advantage of the dynamic structure-based pharmacophore models.

The three pharmacophore models were used to screen the Life Chemicals database consisting of 617822 small molecules. First, we used MODEL_018 with the smallest EF value of the three pharmacophore models to screen the database, and 456541 candidates were hit. Then we used MODEL 011 for screening, and 201270 candidates were hit; finally, we used MODEL_002 for screening, and 98122 candidates were hit. However, when only MODEL 002 was used to screen the database, the number of molecules was 4-fold greater than that using the hierarchical screening method. These results indicated that a high-efficiency hierarchical screening method using pharmacophore models generated by GALAHAD can be used to screen for novel HDACis.

Molecular Docking-Based Virtual Screening of Novel HDAC8 Inhibitors In order to estimate the rationality of the 98122 candidates hit by pharmacophore-based screening and confirm whether they could bind to the active pocket of HDACs, all the candidates were docked into the active site of HDAC8 using a semi-flexible docking method reported in a previously validated protocol. ${ }^{17)}$ When the GA value is 30 , the average RMSD value is $1.08 \AA$ and the docking scores derived from Chemscore and ChemPLP were used for docking. According to the data, 463 small candidates were screened out when we set the cutoff values of the ChemPLP score at $>68.5$ and the Chemscore at $>21.4{ }^{17)}$ Finally, we used SYBYL-X 2.0 for chemical diversity analysis, and 31 hit candidate compounds with different structures were screened out and used for further validation (Supplementary Table 4).

MD-Based Virtual Screening of Hit Compounds with HDAC8 Recently, MD simulation has been used for analyzing biomolecular interactions with the Poisson-Boltzmann surface area (MM-PBSA) method to predict the interactions of druglike compounds with HDACs and analysis the $\triangle G_{\text {bind }}$, and higher $\triangle G_{\text {bind }}$ meant stronger association, ${ }^{16-19)}$ it is an essential validation before drawing conclusions from the docking results. We collected 10 different types of HDACs from the PBD database (1T69, 1T64, 4QA3, 3F07, 3ZNR, 3C10, 4CBY, 4LXZ, 3MAX, and $4 \mathrm{BKX}$ ) for preliminary testing in which the active sites were combined with different crystalline ligands. Moreover, 10 decoys (ZINC06430861, ZINC03413613, ZINC55476972, ZINC09112458, ZINC58202414, ZINC58381831, ZINC02933910, 


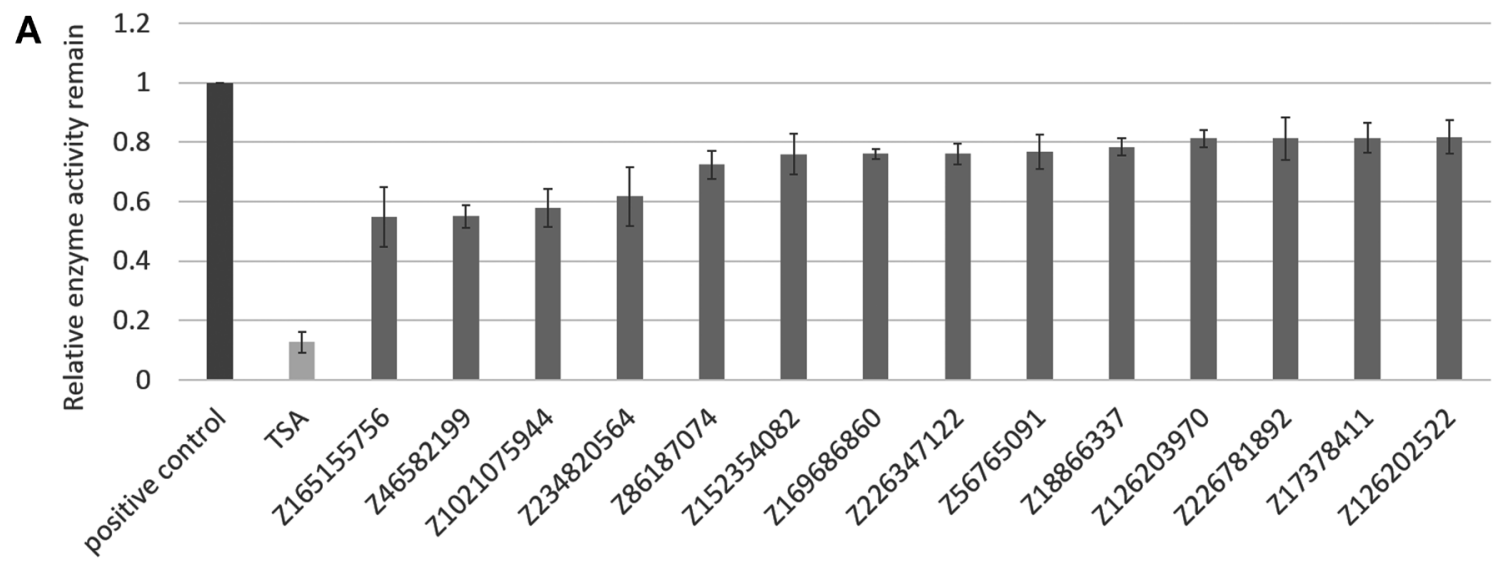

B

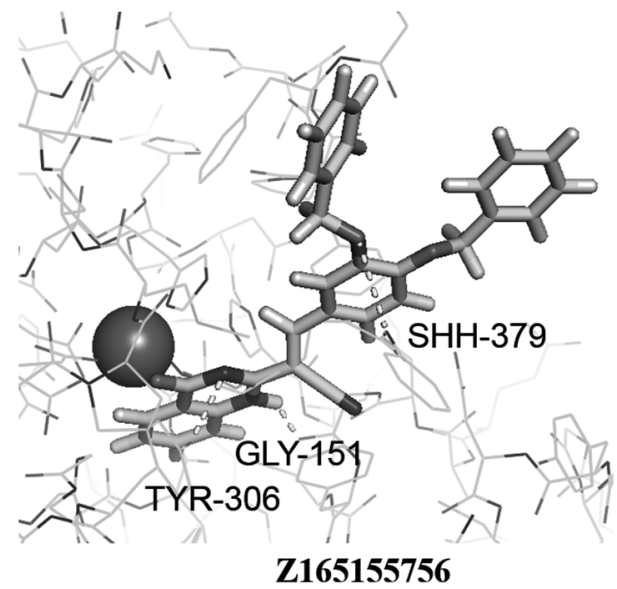

C

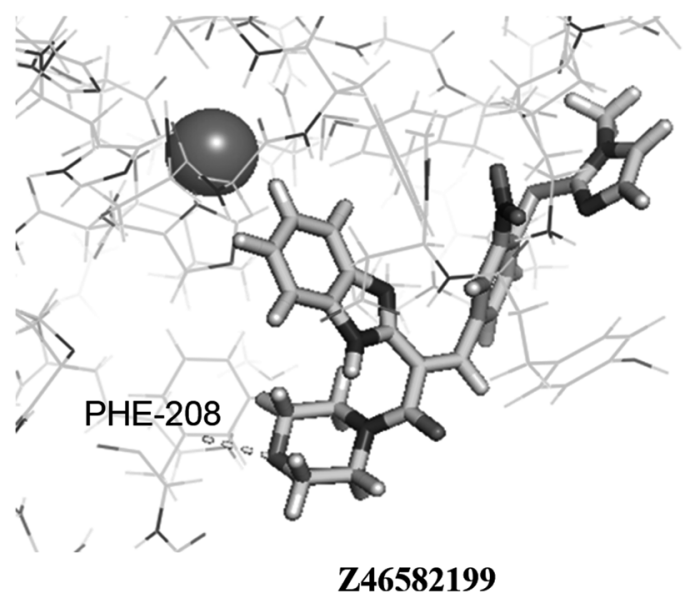

Fig. 2. Inhibitory Effects of the 14 Hit Chemical Candidates against HDACs and Molecular Docking Results of Two Hit Compounds

(A) Inhibitory effects of the hit HDACi. The positive control (DMSO) is shown in gray, the inhibitor control in white gray, and candidate compounds in light gray. Results are expressed as mean \pm S.D. $(n \geq 3)$. ${ }^{*} p<0.05$. (B, C) Docking analysis of Z165155756 and Z46582199 to HDAC. Lines, amino acid residues; spheres, the metal ion $\left(\mathrm{Zn}^{2+}\right)$; white gray dotted line, hydrogen bonds.

ZINC43736119, ZINC08254751, and ZINC10760033) were randomly selected from the MUBD database as decoys. ${ }^{20)}$ The results showed that there was a significant difference between active sites and decoys (Fig. 1B), indicating that potential inhibitors of HDAC may have higher free energy. Therefore, MD simulation could be used to identify new HDACis.

MD simulation was next used to identify effective compounds after docking screening. The $\triangle G_{\text {bind }}$ values of active sites and decoys are listed in Supplementary Table 5. Compounds with $\triangle G_{\text {bind }}$ values $>9.25$ (average of decoys) were selected as effective candidate compounds, and 14 small molecules (Z165155756, Z46582199, Z1021075944, Z234820564, Z86187074, Z152354082, Z169686860, Z226347122, Z56765091, Z18866337, Z126203970, Z226781892, Z17378411, and Z126202522) were identified (Fig. 1C). The hit compounds were purchased from Life Chemicals for further in vitro validation.

Inhibitory Enzyme Activity Evaluation After virtual screening, in vitro experiments were conducted to analyze the enzyme inhibitory activity of the final 14 hit chemical compounds. The results indicated that two compounds, Z165155756 and Z46582199, can significantly inhibit the enzyme activity of HDACs. After treatment with the hit candidates, the relative enzyme activities were $54 \%$ and $55 \%$, respectively, but the other 12 small molecules did not significantly inhibit the activity against HDACs (Fig. 2A).
The structures of the two lead chemical candidates did not belong to either of the four main classes of HDACis, i.e., hydroxamic acids, aliphatic acids, benzamides, and cyclic peptides. ${ }^{21)}$ To investigate the binding modes of the two active candidates further, we analyzed the docking positions of the two active molecules relative to the active site of HDAC8, which showed important interactions between the chemical candidates and active site residues (Fig. 2B). Z165155756 has three functional groups: phenylmethoxy; phenyl; and quinazolinyl. The quinazolinyl group can form covalent chelate complexes with $\mathrm{ZN}^{2+}$ in the active site of HDAC enzymes and lead to reversible inhibition of their biological functions. It can also form hydrogen bond interactions with HDAC residues GLY-151 and TYR-306. Z46582199 has four main functional groups: benzimidazolyl; nitrophenyl; methylimidazole; and morpholino. The morpholino group has hydrophobic contact with HDAC residue PHE208. The two lead chemical candidates were selected for further analysis of their antitumor effects.

Antiproliferative Activity and Apoptosis-Inducing Mechanism HDACis can selectively induce cell cycle arrest and apoptosis in many cancer cells. ${ }^{20)}$ To analyze the cytotoxicity of the candidates Z165155756 and Z46582199, MTT and flow cytometry assays were designed and performed. First, HepG2 and A2780 cells were treated with four different concentrations $(1,10,50$, and $100 \mu \mathrm{M})$ of the two candidate compounds 

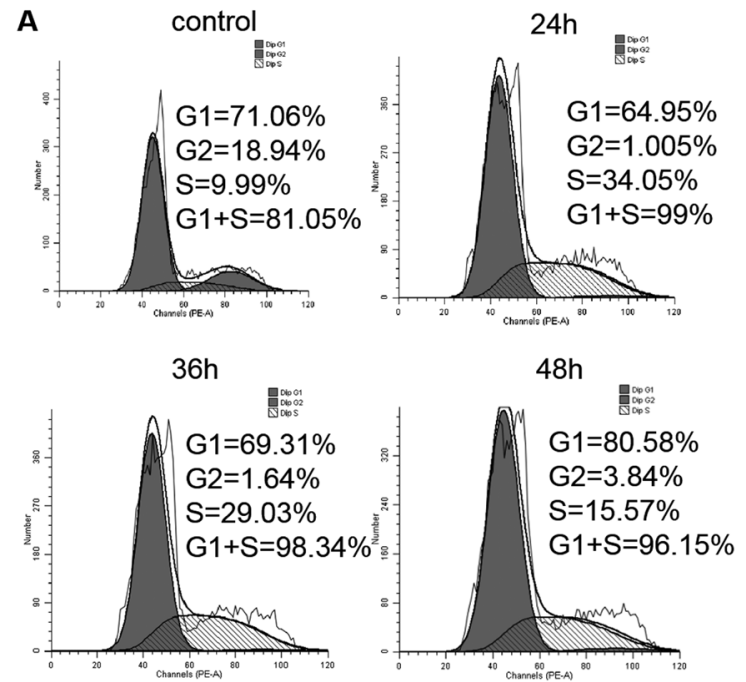

B
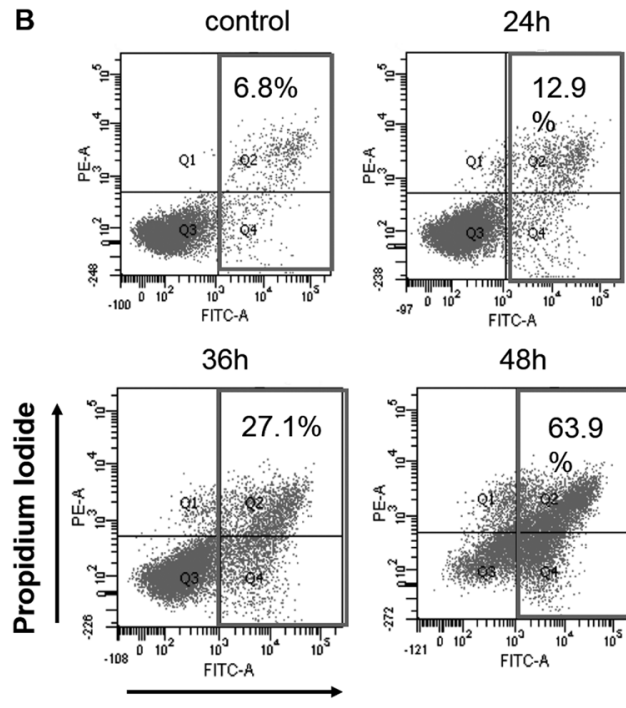

Annexin V-FITC

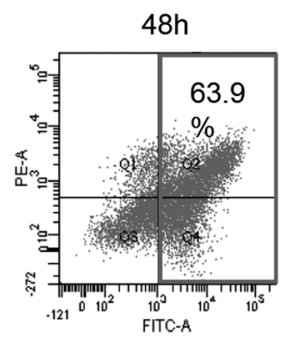

Fig. 3. Z165155756 Induces G1/S Arrest and Apoptosis in A2780 Cells

(A) A2780 cells were incubated with DMSO $(40 \mu \mathrm{M})$ for $48 \mathrm{~h}$ or Z165155756 $(40 \mu \mathrm{M})$ for 24 , 36, or $48 \mathrm{~h}$. Harvested cells were stained with PI, and the cell cycle distribution was analyzed with flow cytometry. (B) A2780 cells were incubated with DMSO $(40 \mu \mathrm{M})$ for $48 \mathrm{~h}$ or Z165155756 $(40 \mu \mathrm{M})$ for 24,36 , or and $48 \mathrm{~h}$. Harvested cells were stained with Annexin V-FITC/PI, and the percentage of apoptotic cells was analyzed with flow cytometry. Three independent experiments were performed, and the results are expressed mean \pm S.D.

Table 1. Comparison of the $\mathrm{IC}_{50}$ Values of Z165155756 and Z46582199 against the A2780, HOSEpiC, HepG2 and L02 Cell Lines

\begin{tabular}{|c|c|c|c|c|}
\hline \multirow{2}{*}{ Chemicals } & \multicolumn{4}{|c|}{$\mathrm{IC}_{50}(\mu \mathrm{M})$} \\
\hline & A2780 & HOSEPIC & HepG2 & L02 \\
\hline Z165155756 & $34.92 \pm 6.22$ & $189.866 \pm 22.07$ & $62.29 \pm 7.54$ & $224.39 \pm 28.12$ \\
\hline Z46582199 & \multicolumn{4}{|c|}{$>300$} \\
\hline
\end{tabular}

for $48 \mathrm{~h}$, after which the $\mathrm{IC}_{50}$ values indicated that $\mathrm{Z} 165155756$ showed stronger inhibitory effects than Z46582199 (Table 1). The $\mathrm{IC}_{50}$ values of Z165155756 against A2780 and HepG2 cells were $34.92 \pm 6.22 \mu \mathrm{M}$ and $62.29 \pm 7.54 \mu \mathrm{M}$, respectively. To determine whether Z165155756 is nontoxic to normal cells, we also treated HOSEpiC and L02 cells with different concentrations of the candidate compounds for $48 \mathrm{~h}$, after which the $\mathrm{IC}_{50}$ values were $189.86 \pm 22.07 \mu \mathrm{M}$ and $224.39 \pm 28.12 \mu \mathrm{M}$, respectively. The $\mathrm{IC}_{50}$ values of $\mathrm{Z} 46582199$ against the two cancer cell lines were greater than $300 \mu \mathrm{M}$, and therefore it may not exert anticancer activity in these cells. These results indicated that Z165155756 shows promising selective inhibitory activity against cancer cell viability, particularly in A2780 cells.

Because Z165155756 significantly inhibited the viability of A2780 cells, we also analyzed the changes after Z165155756 co-culture using flow cytometry. To determine whether the inhibition of cancer cell viability is associated with the induction of cell cycle arrest, A2780 cells were treated with Z165155756 for $0,24,36$, and $48 \mathrm{~h}$ and then stained with PI. The percentage of cells in G1/S phase increased significantly by $17.95 \pm 3.1 \%, 17.29 \pm 2.6 \%$, and $15.1 \pm 4.2 \%$ at 24,36 , and $48 \mathrm{~h}$, respectively (Fig. 3A). In addition to cell cycle arrest, induction of apoptosis is another key mechanism of HDACis. ${ }^{22)}$ The percentage of apoptotic A2780 cells after co-culture with Z165155756 for $0,24,36$, and $48 \mathrm{~h}$ was also examined and found to be $6.8 \pm 1.2,12.9 \pm 2.7,27.1 \pm 5.6$, and $63.9 \pm 4.2 \%$, respectively (Fig. 3B), indicating that Z165155756 induces apoptosis in a time-dependent manner. The results showed that
Z165155756 markedly inhibits the viability of A2780 cells via selective induction of G1/S phase arrest and promotes apoptosis.

\section{Discussion}

We improved a hierarchical virtual screening protocol through the screening of different structure-based pharmacophore models and docking to the active site of HDAC8 to identify drug-like compounds in the Life Chemicals database. Compounds with high docking scores were used in MD simulations to analyze the interactions of hit compounds with HDAC. Potential HDACis were identified based on the $\triangle G_{\text {bind }}$ values. The improved protocol appears to be an essential validation before drawing any conclusions from the pharmacophore and docking results. Based on the improved virtual screening workflow, 14 hit chemical compounds appeared to be potential HDACis. The 14 hit chemical candidate compounds were assessed using SciFinder scholar (https:// scifinder.cas.org/) and the results indicated that they had not previously been assessed for their HDAC-inhibitory effects. The in vitro enzyme inhibition assay confirmed that Z165155756 and Z46582199 have significant HDAC-inhibitory effects. The two lead chemical candidate compounds have lipophilic linker parts and zinc-chelating groups, with the same hydrophobic features and HB acceptors as in the pharmacophore models. Moreover, the two candidate compounds have novel structures that do not belong to the four classes of HDACis, offering opportunities for future HDACi design.

The cytotoxicity assay indicated that, in agreement with 
its inhibitory effects on HDAC, Z165155756 shows promising selective inhibitory activity against cancer cells, particularly A2780 cells. Moreover, Z165155756 significantly inhibited the viability of A2780 cells via selective induction of $\mathrm{G} 1 / \mathrm{S}$ phase arrest and promoted apoptosis. All these results indicate that the method reported here improves the HDACi screening protocol. The method was used to identify the novel structure of the promising HDACi Z165155756. Based on its effects in human ovarian carcinoma cells, Z165155756 is worthy of further investigation.

Acknowledgments This work was supported by the Natural Science Foundation of Jilin Province, P. R. China (No. 20180101242JC), Natural Nature Science Foundation of China (81700709), Research Foundation of Jilin Provincial Science\&Technology Development (20180520105JH), and Systems Biology Research on Genome and Transcriptome of Stem Cells (2017030) of Jilin Province Sunbird Regenerative Medical Engineering Co., Ltd.

Conflict of Interest The authors declare no conflict of interest.

Supplementary Materials The online version of this article contains supplementary materials.

\section{References}

\begin{tabular}{ll} 
1) & West A. C., Johnstone R. W., J. Clin. Invest., 124, 30-39 (2014). \\
\hline 2) & Lane A. A., Chabner B. A., J. Clin. Oncol., 27, 5459-5468 (2009). \\
\hline 3) & Witt O., Deubzer H. E., Milde T., Oehme I., Cancer Lett., 277, 8-21 \\
\hline 4) & Da09). \\
5) & Ellison L., Atadja P. W., Johnstone R. W., Mol. Cancer Ther., 8, \\
& 1409-1420 (2009).
\end{tabular}
6) Marks P. A., Xu W. S., J. Cell. Biochem., 107, 600-608 (2009).

7) Chun P., Arch. Rch. Pharm. Res., 38, 933-949 (2015).

8) Kelly W. K., O'Connor O. A., Marks P. A., Expert Opin. Investig. Drugs, 11, 1695-1713 (2002).

9) Durrant J. D., McCammon J. A., BMC Biol., 9, 71 (2011).

10) Hou X., Du J., Liu R., Zhou Y., Li M., Xu W., Fang H., J. Chem. Inf. Model., 55, 861-871 (2015).

11) Liu X., Shi D., Zhou S., Liu H., Liu H., Yao X., Expert Opin. Drug Dis., 13, 23-37 (2018).

12) Lu W., Zhang R., Jiang H., Zhang H., Luo C., Front. Chem., 6, 57 (2018).

13) Kumari R., Kumar R., Lynn A., J. Chem. Inf. Model., 54, 19511962 (2014)

14) Wang D. F., Helquist P., Wiech N. L., Wiest O., J. Med. Chem., 48, 6936-6947 (2005)

15) Kollman P. A., Massova I., Reyes C., Kuhn B., Huo S., Chong L. Lee M., Lee T., Duan Y., Wang W., Donini O., Cieplak P., Srinivasan J., Case D. A., Cheatham T. E. III, Acc. Chem. Res., 33, 889-897 (2000).

16) Liu C. J., Zhang X. L., Luo D. Y., Zhu W. F., Wan H. F., Yang J. P., Yang X. J., Wan F. S., Asian Pac. J. Cancer Prev., 16, 741-746 (2015).

17) Huang Y. X., Zhao J., Song Q. H., Zheng L. H., Fan C., Liu T. T., Bao Y. L., Sun L. G., Zhang L. B., Li Y. X., BMC Pharmacol. Toxicol., 17, 32 (2016).

18) Xia J., Tilahun E. L., Kebede E. H., Reid T. E., Zhang L., Wang X. S. J., Chem. Inf. Model., 55, 374-388 (2015).

19) Ferrari A. M., Degliesposti G., Sgobba M., Rastelli G., Bioorg. Med. Chem., 15, 7865-7877 (2007).

20) Brice A. R., Dominy B. N., J. Comput. Chem., 32, 1431-1440 (2011).

21) New M., Olzscha H., La Thangue N. B., Mol. Oncol., 6, 637-656 (2012).

22) Giannini G., Cabri W., Fattorusso C., Rodriquez M., Rodriquez M. Future Med. Chem., 4, 1439-1460 (2012). 